\title{
Late side effects of radiation treatment for head and neck cancer
}

\author{
Itzhak Brook \\ Department of Pediatrics, Georgetown University School of Medicine, Washington, DC, USA
}

Received: April 14, 2020

Revised: June 6, 2020

Accepted: June 9, 2020

Correspondence:

Itzhak Brook

Department of Pediatrics,

Georgetown University School of

Medicine, 4431 Albemarle St NW,

Washington, DC 20016, USA

Tel: +1-202-363-4253

E-mail: ib6@georgetown.edu

ORCID:

https://orcid.org/0000-0001-6068-1475
Patients undergoing radiation therapy for head and neck cancer (HNC) experience significant early and long-term side effects. The likelihood and severity of complications depends on a number of factors, including the total dose of radiation delivered, over what time it was delivered and what parts of the head and neck received radiation. Late side effects include: permanent loss of saliva; osteoradionecrosis; radiation recall myositis, pharyngoesophageal stenosis; dental caries; oral cavity necrosis; fibrosis; impaired wound healing; skin changes and skin cancer; lymphedema; hypothyroidism, hyperparathyroidism, lightheadedness, dizziness and headaches; secondary cancer; and eye, ear, neurological and neck structures damage. Patients who undergo radiotherapy for nasopharyngeal carcinoma tend to suffer from chronic sinusitis. These side effects present difficult challenges to the patients and their caregivers and require life-long strategies to alleviate their deleterious effect on basic life functions and on the quality of life. This review presents these side effects and their management.

Keywords: Radiation, Side effects of radiation, Fibrosis, Head and neck cancer, Lymphedema, Mucositis

\section{Introduction}

Radiation therapy (RT) is often used to treat head and neck cancer (HNC) [1]. RT can damage blood vessels that nourish muscles, nerves, and bones resulting in a progressive "radiation fibrosis syndrome", which causes a variety of complications [2]. The likelihood and severity of complications depends on a number of factors, including the total dose of radiation delivered, over what time it was delivered and what parts of the head and neck received radiation. The side effects of RT for HNC are divided into early (acute) and long-term (chronic) effects [3]. Early side effects occur during the course of therapy and during the immediate post therapy period (approximately 2-3 weeks after the completion of a course of RT). Late effects can manifest any time thereafter, from weeks to years later [4].

Patients are usually most bothered by the early effects of RT, although these will generally resolve over time. Knowledge of the ra- diation side effects can allow their early detection and proper management. Late side effects include permanent loss of saliva; osteoradionecrosis; pharyngoesophageal stenosis; dental caries; oral cavity necrosis; fibrosis; radiation recall myositis, impaired wound healing; skin changes and skin cancer; lymphedema; hypothyroidism, hyperparathyroidism, lightheadedness, dizziness and headaches; secondary cancer; and eye, ear, neurological and neck structures damage. This manuscript reviews the major late side effects of RT and their management (Table 1).

\section{Permanent Dry Mouth (Xerostomia)}

Although xerostomia and thick saliva improves in most people, they can be long lasting and affects quality of life. RT can lead to irreversible salivary glands cells damage $[2,5]$. Serous salivary glands (parotid \& submandibular) are very sensitive to radiation. RT often leads to marked changes in the quantity and quality of saliva

Copyright@ 2020 The Korean Society for Radiation Oncology

This is an Open Access article distributed under the terms of the Creative Commons Attribution Non-Commercial License (http://creativecommons.org/licenses/by-nc/4.0/) which permits unrestricted non-commercial use, distribution, and reproduction in any medium, provided the original work is properly cited. 


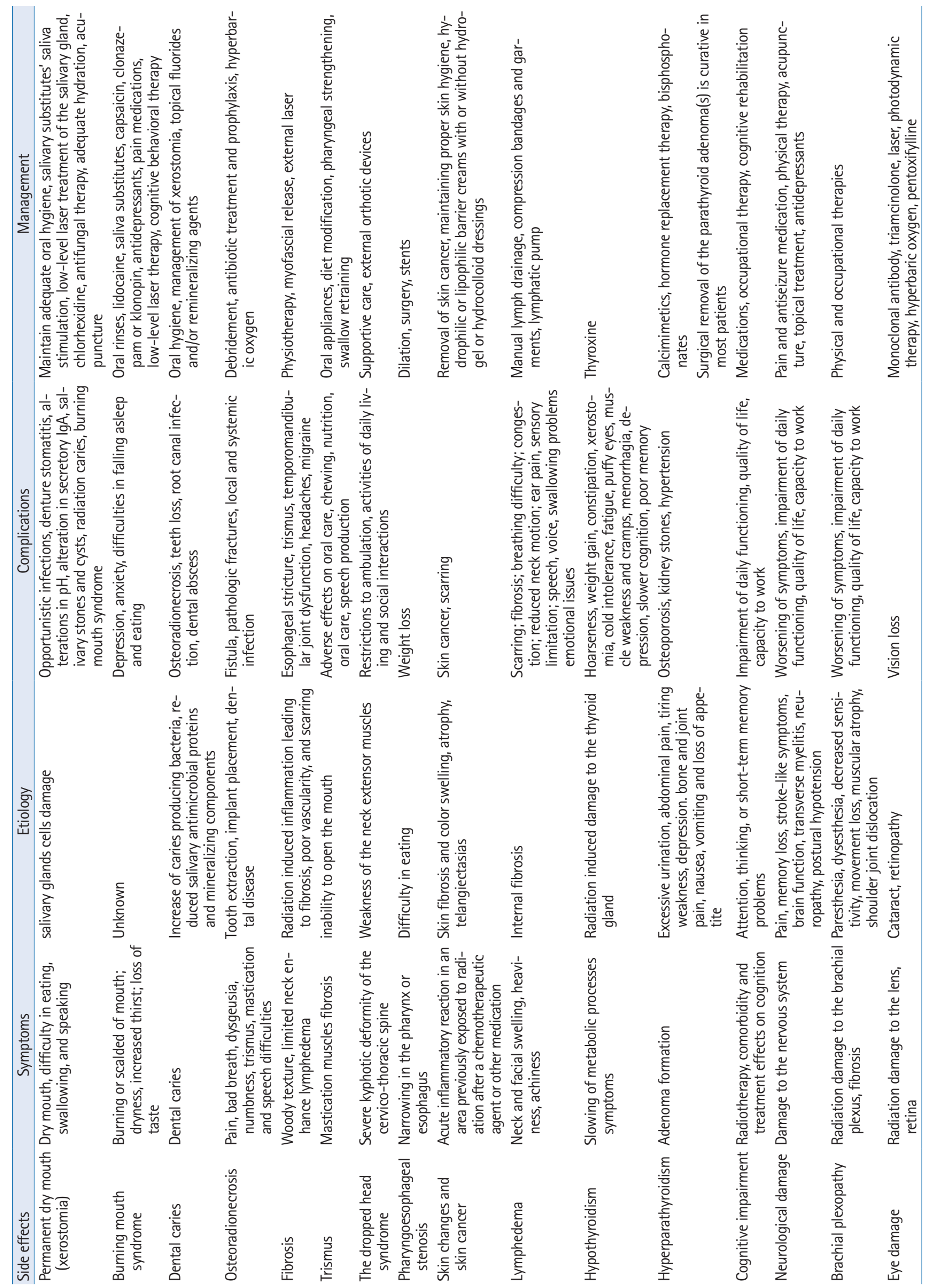


altering its consistency from watery viscous. Xerostomia can lead to opportunistic infections (mostly fungal), denture stomatitis, alterations in $\mathrm{pH}$, alteration in secretory $\lg \mathrm{A}$, salivary stones and cysts, radiation caries (subgingival), and burning mouth syndrome [6]. It can lead to difficulty in eating, swallowing, and speaking, increase the risk of cavities and dental disease, and complicates dentures maintenance. Patients with xerostomia should maintain adequate oral hygiene to minimize the risk of oral lesions. Periodontal disease can be accelerated and caries can become rampant unless preventive measures are instituted.

Management and prevention include [7,8]: (1) palliative use of salivary substitutes (gels, rinses), (2) non-pharmacological saliva stimulation, (3) salivary stimulants, (4) low-level laser treatment of the salivary glands, (5) prophylactic chlorhexidine, (6) antifungal therapy, (7) preventing thrush, and (8) drinking plenty of liquids. These measures can help in coping with xerostomia: drinking adequate fluids; frequent sipping or mouth spraying with water; sucking on ice chips and/or sugar-free popsicles; consuming acidic or bitter substances, using sugarless gum and sugarless hard candy, and rinsing and gargling with diet ginger ale or a weak solution of salt and baking soda are helpful to refresh the mouth, loosen thick oral secretions, and alleviate mild pain [9].

The use of saliva substitutes or artificial saliva (containing hyetellose, hyprolose, or carmellose), stimulation of saliva production from intact salivary glandular tissues by taste/mastication, pharmacological; avoiding smoking and all products that contain caffeine or alcohol, using a bedside humidifier at night, and raising the head of the bed can be helpful. Low-level laser treatment of the salivary glands can intensify cell metabolism and its application on salivary glands could improve salivation. Treatment include salivary stimulants (sialagogues), such as pilocarpine, amifostine, and cevimeline [10]. Preliminary data suggest that hyperbaric oxygen (HBO) can provides benefit for patients with xerostomia who have some residual salivary gland function $[9,11]$. Acupuncture has been found helpful in reducing pain and dry mouth [12]. Dietary change from dry, tough food to easier to swallow moist, softer ones can improve nutritional status and quality of life. Use of a humidification especially in the bedroom can provide relief.

\section{Burning Mouth Syndrome}

Chronic or recurrent mouth burning caused by the radiation and enhanced by xerostomia is termed secondary "burning mouth syndrome" [13]. Symptoms may include: a burning or scalded sensation affecting the tongue, lips, gums, palate, throat or whole mouth; mouth dryness and increased thirst; and loss of taste or changes in taste (e.g., bitter or metallic). The mouth discomfort can be present throughout the day or slowly worsen. It may last for months to years. Symptoms may suddenly disappear or rarely become less frequent. Eating or drinking can bring temporarily relief. The discomfort can lead to depression, anxiety, and difficulties in falling asleep and eating. Avoiding acidic foods, spicy foods and carbonated beverages, tobacco, and excessive stress may help.

Treatment is symptomatic and include: specific oral rinses or lidocaine, saliva substitutes, capsaicin, clonazepam or klonopin, antidepressants, pain medications, low-level laser therapy, and cognitive behavioral therapy [13].

\section{Dental Caries}

Factors enhancing the occurrence of caries after RT include the increase in the number of oral caries producing bacteria (Streptococcus mutans and Lactobacillus species), reduced concentrations of salivary antimicrobial proteins, and loss of saliva's mineralizing components [14].

Treatment strategies include optimal oral hygiene and management of xerostomia. Resistance to caries can be enhanced by using topical fluorides and/or remineralizing agents (high in calcium phosphate and fluoride) using dental trays [14]. Topical fluorides or chlorhexidine rinses may reduce $S$. mutans levels but not Lactobacilli. Because of adverse drug interactions, fluoride and chlorhexidine dosing should be separated by several hours [14].

\section{Osteoradionecrosis}

Osteoradionecrosis can necessitate surgical intervention and reconstruction [15]. Depending on its location and extent, symptoms may include pain, bad breath, dysgeusia, numbness, trismus, mastication and speech difficulties, fistula, pathologic fractures, and local, spreading, or systemic infection. Osteoradionecrosis is a lifelong risk for those who have received high-dose radiation [16].

The mandible is the most frequently affected bone, especially in those treated for nasopharyngeal cancer. Maxillary involvement is rare because of the collateral blood circulation it receives. Tooth extraction and dental disease in irradiated areas are major contributors to osteoradionecrosis. It may be necessary to remove decayed teeth before RT if they are in the area receiving RT [16]. Ideally, at least 7 to 14 days should be allowed for healing before initiation of RT; some have suggested allowing up to 21 days. Oral disease should be treated prior to receiving radiation therapy whenever possible [15].

Mild osteoradionecrosis can be conservatively treated with debridement, antibiotics, and occasionally ultrasound. Topical antibiotics (e.g., tetracycline) or antiseptics (e.g., chlorhexidine) may contrib- 
ute to wound resolution. Exposed bone should be covered with mucosa and necrotic bone removed. When necrosis is extensive, radical resection, followed by microvascular reconstruction is often used. The combination of pentoxifylline and tocopherol was effective in treating bisphosphonate and radiation related osteonecrosis of the jaw [17]. The prophylactic use of the combination reduced the incidence of osteoradionecrosis following dental extraction [15]. HBO has been often used in patients at risk or those who develop osteoradionecrosis. However, the available data are conflicting about the clinical benefit of HBO for prevention and therapy [18].

Patients should inform their dentists about their RT prior to extraction or dental surgery including implant placement [17]. Osteonecrosis may be prevented by administration of a series of $\mathrm{HBO}$ therapy before and after these procedures. This is recommended if the involved tooth is in an area that has been exposed to a high dose of radiation. Dental prophylaxis can reduce the risk of osteoradionecrosis. Special fluoride treatments may help with dental problems along with brushing, flossing, and regular cleaning by a dental hygienist [18].

\section{Fibrosis}

Fibrosis can develop in the skin, subcutaneous tissue, muscles, or other organs, depending upon the treatment site $[3,4]$. It generally starts eight to twelve weeks after the initiation of RT and is a lifelong issue. Radiation to the neck can cause woody texture and limited mobility of the neck, enhance lymphedema significant tightness to of the neck and shoulders muscles including the scalenes, trapezius, and sternocleidomastoid muscles. Fibrosis may cause both cosmetic and functional impairment, leading to deterioration in the quality of life. Fibrosis can also occur in the pharynx and esophagus, leading to stricture and temporomandibular joint problems including mandibular dysfunction. Patients can be assisted by mandibular stretching exercises and the use of prosthetic aids [19].

Interventions should be instituted before trismus develops. If clinically significant changes develop, several approaches can be considered, including stabilization of occlusion and use of trigger-point injection and other pain management strategies, muscle relaxants, and tricyclic medications.

To minimize the risk of neck tightness, patients should maintain flexibility of the neck muscles by stretching exercises including chin curls, head rotations, shoulder shrugs, and shoulder circles. Exercise can reduce neck tightness and increases the range of neck motion. These exercises should be performed throughout life. After fibrosis has developed individuals may benefits from myofascial release (MFR) [4]. MFR is a hands-on method of massaging and stretching the connective tissue of the head/neck to increase range of motion, increase flexibility, decrease pain, and improve posture. Treatment can also break down the fibrosis. The earlier the intervention, the better it is for the patient.

Muscle tightness can trigger headaches, leading to migraines. The mastication muscles are also often involved. Treatment of muscle fibrosis can often alleviate and reduce the frequency of such headaches [20]. Fibrosis can become more extensive after surgery or re-radiation.

\section{Trismus}

Trismus or lockjaw is common following radiation targeting the base of tongue, tonsil, retromolar trigone, soft palate, masseter muscles, pterygoid muscles, and the temporomandibular joint (TMG) [21]. The prevalence of trismus increases with increasing radiation dose, and levels in excess of $60 \mathrm{~Gy}$ are more likely to cause it [21].

Mastication muscles fibrosis can lead to progressive inability to open the mouth. Trismus can adversely affect proper oral care and treatment, chewing, nutrition, oral care, speech production, and intubation. Prophylactic trismus prevention exercises can be initiated in those considered at high risk [2,4]. If aggressive intervention is needed, a speech pathologist may recommend prophylactic use of tongue blade therapy or a device (e.g., TheraBite, OraStretch, Dynasplint) [22]. A wide array of appliances is available for the treatment of trismus [23]. Swallowing dysfunction often requires a change in diet, pharyngeal strengthening, or swallow retraining, especially in those who have had surgery and/or chemotherapy.

\section{The Dropped Head Syndrome}

The dropped head syndrome (DHS) is a disabling condition caused by severe weakness of the neck extensor muscles causing progressive reducible kyphosis of the cervical spine and the inability to hold the head up [24]. DHS can occur from 3 months to 30 years after RT.

Treatment with physiotherapy and surgery have not been very successful, and the management of DHS is supportive, including employing a cervical collar to maintain the head in an upright position $[4,24]$. The condition generally does not spread to other muscles or worsens.

\section{Pharyngoesophageal Stenosis}

Pharyngoesophageal stenosis can be a delayed complication of RT [25]. Pharyngoesophageal (PE) stenosis is an area of narrowing in the pharynx or esophagus. This stenosis can make eating difficult, 
particularly solid food. If the PE segment closed off, the patient is not able to eat or drink by mouth and needs gastric tube. Treatment includes frequent placement of dilating catheters to stretch open the narrowed segment or by surgically removing the blocked segment followed by flap reconstruction [25].

\section{Skin Changes and Skin Cancer}

Patients can experience "radiation recall dermatitis" [26]. Patients who had early severe dermatitis, may experience inflammatory waves occurring weeks to years later [27]. This is characterized by a skin rash typified by redness, swelling, and/or skin blistering. The rash is often painful resembling severe sunburn.

Late-stage or "chronic radiation dermatitis" typically presents months to years after RT. It is characterized by skin fibrosis, slight color changes to the skin or mild swelling, atrophy, and telangiectasias. Individuals generally lose hair in the region that received radiation. Radiation can increase the risk for skin cancers (basal cell and squamous cell carcinoma) [4]. Regular follow-up by a dermatologist is important.

\section{Lymphedema}

Lymphedema can cause chronic inflammation and reactive fibrosis of the affected tissues. RT creates scarring which interferes with the function of the lymphatics resulting in slow lymphatic swelling. Lymphedema generally starts and progresses slowly 8 to 12 weeks after initiation of RT and can be a life-long issue $[4,5]$.

Lymphedema can be external (skin and soft tissue) and internal (pharyngeal and laryngeal mucosa). Lymphedema causes heaviness and achiness sensations, and may lead to skin changes.

Lymphedema has several stages [28]:

- Stage 0: No swelling, but a sense of heaviness in the neck.

-Stage 1a: Visible mild swelling without pitting. Reversible.

-Stage 1b: Visible mild swelling with pitting. Reversible.

-Stage 2: Firm-pitting swelling that is irreversible. No visible tissue changes.

- Stage 3: Irreversible tissue changes with scarring and fibrosis.

Lymphedema can cause difficulty in breathing, congestion, impairment in vision, motor (reduced neck motion, jaw tightness or trismus, and chest tightness), ear pain, and sensory limitations, speech, voice, and swallowing problems (i.e., inability to use an electrolarynx, difficulty in articulation, drooling, and loss of food from mouth), and emotional issues (depression, frustration, and embarrassment) [28].

Over time, the lymphatics find newer way of drainage, and the swelling and neck tightness generally goes down. Sleeping with the upper body in an elevated position can use gravity to speed the process of lymph fluid drainage. Specialists in reducing edema can assist the patient to enhance the drainage and shortening the time for the swelling to decrease. This treatment can also prevent permanent swelling and fibrosis.

Treatment of lymphedema includes [28]: (1) manual lymph drainage (face and neck, deep lymphatics, trunk, intra oral), (2) compression bandages and garments, (3) use of tactile lymphatic pump, (4) remedial exercises, (5) skin care, (6) elastic therapeutic tape (Kinesio Tape), and (7) oncology rehabilitation [2,4]. Diuretics, surgical removal (debulking), liposuction, compression pumps, and elevation of the head alone are ineffective treatments [28].

\section{Hypothyroidism}

Most individuals receiving RT develop hypothyroidism. The symptoms of hypothyroidism vary; some individuals have no symptoms while others have dramatic or, rarely, life-threatening symptoms [29]. Most symptoms of hypothyroidism are due to the slowing of metabolic processes.

Thyroid deficiency can be corrected by taking synthetic thyroid hormone (thyroxine) $[4,29]$. The patient should be reevaluated and serum thyroid stimulating hormone (TSH) should be measured in 3 to 6 weeks, and the dose adjusted if needed. The process of adjusting the dose of hormone every 3 to 6 weeks is continued, based upon periodic measurements of TSH until it returns to normal. After identification of the proper maintenance dose, TSH levels should be measured at least yearly.

\section{Hyperparathyroidism}

The parathyroid glands are resistant to RT. However, hyperparathyroidism (HPT) due to adenoma formation can occurs in individuals who had received RT for HNC after a longer latency period [30]. Signs and symptoms of HPT include: osteoporosis, kidney stones, excessive urination, abdominal pain, tiring easily or weakness, depression and forgetfulness, bone and joint pain, frequent complaints of illness with no apparent cause, nausea, vomiting and loss of appetite [30]. HPT is diagnosed by finding elevated calcium levels in the blood, bone mineral density test (bone densitometry), a 24-hour collection of urine, and imaging tests of kidneys.

Treatment includes watchful waiting in those with normal calcium levels and kidney functions, and normal bone density. Medications to treat HPT include calcimimetics, hormone replacement therapy to retain calcium, and bisphosphonates. Surgical removal of the parathyroid adenoma(s) is curative in most patients [30]. 


\section{Attention, Thinking, and Memory Problems (Cognitive Problems)}

Many patients who received RT to the head and neck and/or chemotherapy experience attention, thinking, or short-term memory problems [31]. Other causes for cognitive problems are pain, side effects of medications, emotional state, and other medical problems. Cognitive problems can manifest in the following symptoms or behavioral changes: (1) trouble concentrating, focusing, or paying attention, (2) mental fog or disorientation, (3) difficulty with spatial orientation, (4) memory loss or difficulty remembering things, especially names, dates, or phone numbers, (5) problems with understanding, (6) difficulties with judgment and reasoning, and (7) impaired ability to calculate and organize, and impaired language skills. These include difficulties to organize one's thoughts, find the right word, or balance a checkbook: (1) problems in multitasking, (2) processing information slower, (3) behavioral and emotional changes, such as irrational behavior, mood swings, inappropriate anger or crying, and socially inappropriate behavior, and (4) severe confusion.

Management of these cognitive problems includes: (1) medications, including stimulants, cognition-enhancers, antidepressants, narcotics blockers, (2) occupational therapy and vocational rehabilitation, and (3) cognitive rehabilitation and cognitive training.

\section{Neurological Damage}

These can include serious problems such as memory loss, strokelike symptoms, and brain function [31]. RT to the neck can affect the spinal cord, resulting in a self-limited transverse myelitis, known as "Lhermitte's sign". The patient notes an electric shocklike sensation mostly felt with neck bending (flexion) [32]. This condition rarely progresses to a true transverse myelitis which is associated with Brown-Séquard syndrome. RT may cause neuropathy due to nerve injury, although it may take several years for symptoms to appear $[2,4]$. It can also cause peripheral nervous system dysfunction resulting from external compressive fibrosis of soft tissues and reduced blood supply caused by fibrosis. Symptoms depend on which nerves are affected and include: change in sensation, especially in the hands and feet (e.g., numbness, tingling, or pain), muscle weakness (i.e., myopathy), paralysis and/or paresis (weakness) of the diaphragms (phrenic nerve damage), and changes in organ function (i.e., constipation, dizziness) [33].

Acupuncture treatment may improve peripheral neuropathy [34].

Damage to the peripheral and autonomic nervous system can lead to dizziness when standing up from sitting up from sitting or lying down due to postural hypotension.

\section{Brachial Plexopathy}

Radiation-induced brachial plexopathy is caused by radiation damage to the brachial plexus $[2,4,35]$ that provide sensation and muscular innervation for the whole hand. Symptoms include paresthesia, dysesthesia, decreased sensitivity, partial loss of movement, complete paralysis of the arm, muscular atrophy, impaired mobility and partial dislocation of the shoulder joint. The damage to the brachial plexus results from a combination of direct RT nerve damage, the development of fibrosis and damage to blood vessels supplying these nerves. The extent of damage is associated with the radiation dose and technique, and concurrent chemotherapy.

Most develop symptoms within the 3 years (range, 6 months to 20 years) [35]. Rehabilitation includes physical and occupational therapies [33].

\section{Eye Damage}

Radiation can cause sub-capsular and cortical cataract [4]. The risk of lens opacities exists above does of 0.5 Gy. "Radiation retinopathy" is dose related (especially > $45 \mathrm{~Gy}$ ) and is common after RT for nasopharyngeal, paranasal sinus or orbital tumors. Shielding of ocular structures during RT and hyperfractionation can decreased its incidence [36].

Treatment includes intravitreal injection of humanized monoclonal antibody to vascular endothelial growth factor (bevacizumab), intravitreal triamcinolone acetonide, grid macular laser photocoagulation, sector scatter and panretinal laser photocoagulation, photodynamic therapy, HBO and oral pentoxifylline [36]. Advanced proliferative radiation retinopathy complicated by vitreous hemorrhage and/or tractional retinal detachment may require vitrectomy.

\section{Damage to the Ear (Ototoxicity) and Hearing Loss}

Patients who undergo RT for HNC can develop hearing especially when receiving 60 Gy. Complaints include ear heaviness, earache, decreased hearing, tinnitus, and dizziness. Dose of radiation is directly proportional to ototoxicity [37].

Radiation to the ears may result in serous otitis (otitis with effusion) [38]. It is associated with fluid collection in the middle ear and temporary reduced hearing. Serous otitis and conductive deafness are reversible over time. High doses of radiation can cause sensorineural hearing loss due to damage to the inner ear, the auditory nerve, or the vestibular apparatus. This condition is not reversible. Damage to the vestibular apparatus can cause dizziness and vertigo. 


\section{Lightheadedness, Dizziness, and Headaches}

Damage to the peripheral and autonomic nervous system and the carotid artery baroreceptors can lead to dizziness due to orthostatic hypotension [39]. This can be prevented by standing up slowly, wearing of compression stockings, exercising, and keeping well hydrated. The perception of the body's position is determined by the brain by integrating information from the middle ear, eyes, and the body's muscles and joints. The perception of lightheadedness and dizziness after RT may be generated in some individuals by misinformation sent to the cerebellum from the fibrotic neck muscles and damaged middle ear [39].

Dizziness and lightheadedness can be treated by vestibular rehabilitation and exercises that stretch the fibrotic muscles, reduce neck stiffness, and increase the head and neck range of motion [40]. Muscle tightness and fibrosis can trigger headaches leading to migraine. The muscles of mastication are also often involved. Treatment of muscle fibrosis can alleviate and reduce the frequency of headaches [4].

\section{Damage to Neck Structures}

RT can cause lymphedema and fibrosis, carotid artery (CA) stenosis and stroke, CA rupture, oropharyngo-cutaneous fistula, and CA baroreceptors damage leading to permanent and paroxysmal (sudden and recurrent) hypertension [41]. Factors contributing to increased risk of ischemic stroke including CA stenosis and increased deposition of plaque, as well as other pre-existing risk factors for cerebrovascular disease.

Stenosis, and rarely CA rupture [42]. Yearly screening neck ultrasound can lead to early diagnosis. The incidence of CA stenosis is 18\%-38\% in irradiated patients compared to $0 \%-9.2 \%$ in unirradiated patients [43]. CA disease can cause strokes and transient ischemic attack (TIA). It is important to diagnose carotid stenosis or impending rupture early, before a stroke or severe bleeding has occurred. Stenosis can be diagnosed by hearing a bruit sound over the $C A$, ultrasound, $C T$, magnetic resonance angiogram (MRA), MRI, and angiography. Treatment of stenosis caused by RT is usually by placing a stent. Other methods include removal of the blockage (endarterectomy), and prosthetic carotid bypass grafting [44]. RT can damage the CA baroreceptors. These baroreceptors regulate the blood pressure sending messages to the central nervous system to alter the peripheral vascular resistance and cardiac output. Some individual treated with radiation "develop low, labile or paroxysmal hypertension" [45].

Low blood pressure can be cause by damage to the peripheral and autonomic nervous system and the carotid baroreceptors. This can lead to baroreceptor failure manifested by orthostatic hypotension characterized by dizziness when standing up from a sitting or lying down. It can be managed by standing up slowly, wearing of compression stockings, exercising and by keeping well hydrated $[41,45,46]$.

Labile hypertension is characterized by sometime asymptomatic blood pressure fluctuations from low (e.g., 120/80 mmHg) to high (e.g., 170/105 mmHg). A relationship between blood pressure elevation and stress or emotional distress is usually present [47].

Paroxysmal hypertension is characterized by sudden elevation of blood pressure ( $>200 / 110 \mathrm{mmHg}$ ) associated with an abrupt onset of distressful physical symptoms, such as headache, chest pain, dizziness, nausea, palpitations, flushing, and sweating. Episodes can last from 10 minutes to several hours and may occur in different frequency-varying from once or twice daily to once every few months. Between episodes, the blood pressure is normal or may be mildly elevated. Patients generally cannot identify obvious psychological factors that cause the paroxysms [48]. Direct massage of the carotid artery during Doppler ultrasound can lead to such episodes [49]. Medical conditions that can also cause such blood pressure swings need to be excluded (e.g., pheochromocytoma). Both of these conditions are serious and should be treated. Management can be difficult and should be done by experienced specialists.

\section{Secondary Cancers}

RT can rarely result in new local and systemic cancers appearing. The risk is proportional to the administered radiation dose [50]. The secondary cancer can be different from the original and could include local cancers such as skin, mediastinal, oral and thyroid cancer, and systemic cancers such as lymphomas, sarcomas and leukemia [4]. It is important to be closely followed to detect secondary malignancies.

\section{Conclusions}

RT for the HNC causes significant long-term side effects in most patients [5]. Many of these side effects present difficult challenges to the patients and their caregivers. Recognizing and treatment of these side effects can significantly improve the patients' health, long-term survival and quality of life.

\section{Conflict of Interest}

No potential conflict of interest relevant to this article was reported. 


\section{References}

1. Morgan HE, Sher DJ. Adaptive radiotherapy for head and neck cancer. Cancers Head Neck 2020;5:1.

2. Tolentino Ede $S$, Centurion BS, Ferreira LH, Souza AP, Damante $\mathrm{JH}$, Rubira-Bullen IR. Oral adverse effects of head and neck radiotherapy: literature review and suggestion of a clinical oral care guideline for irradiated patients. J Appl Oral Sci 2011; 19:448-54.

3. Stubblefield MD. Clinical evaluation and management of radiation fibrosis syndrome. Phys Med Rehabil Clin N Am 2017;28:89100.

4. Sroussi HY, Epstein JB, Bensadoun RJ, et al. Common oral complications of head and neck cancer radiation therapy: mucositis, infections, saliva change, fibrosis, sensory dysfunctions, dental caries, periodontal disease, and osteoradionecrosis. Cancer Med 2017;6:2918-31.

5. Brook I. My voice [Internet]. [place unknown]: [publisher Blogger]; c2020 [cited 2020 Jun 10]. Available from: https://dribrook. blogspot.com/.

6. Pedersen AM, Sorensen CE, Proctor GB, Carpenter GH, Ekstrom J. Salivary secretion in health and disease. J Oral Rehabil 2018; 45:730-46.

7. Wu WW, Leung KY. A Review on the assessment of radiation induced salivary gland damage after radiotherapy. Front Oncol 2019:9:1090.

8. Gupta N, Pal M, Rawat S, et al. Radiation-induced dental caries, prevention and treatment: a systematic review. Natl J Maxillofac Surg 2015;6:160-6.

9. Ma SJ, Rivers Cl, Serra LM, Singh AK. Long-term outcomes of interventions for radiation-induced xerostomia: a review. World J Clin Oncol 2019;10:1-13.

10. Gil-Montoya JA, Silvestre FJ, Barrios R, Silvestre-Rangil J. Treatment of xerostomia and hyposalivation in the elderly: a systematic review. Med Oral Patol Oral Cir Bucal 2016;21:e355-66.

11. Cheng $\mathrm{CO}_{1} \mathrm{Xu} \mathrm{H}$, Liu L, et al. Efficacy and safety of pilocarpine for radiation-induced xerostomia in patients with head and neck cancer: a systematic review and meta-analysis. J Am Dent Assoc 2016;147:236-43.

12. Zhuang $L_{1}$ Yang $Z_{\text {, Zeng }} X_{1}$ et al. The preventive and therapeutic effect of acupuncture for radiation-induced xerostomia in patients with head and neck cancer: a systematic review. Integr Cancer Ther 2013;12:197-205.

13. Fischoff DK, Spivakovsky S. Little evidence to support or refute interventions for the management of burning mouth syndrome. Evid Based Dent 2017;18:57-8.

14. Muller VJ, Belibasakis GN, Bosshard PP, et al. Change of saliva composition with radiotherapy. Arch Oral Biol 2019;106:104480.

15. Kojima Y, Yanamoto S, Umeda M, et al. Relationship between dental status and development of osteoradionecrosis of the jaw: a multicenter retrospective study. Oral Surg Oral Med Oral Pathol Oral Radiol 2017;124:139-45.

16. Seo MH, Eo MY, Myoung H, Kim SM, Lee JH. The effects of pentoxifylline and tocopherol in jaw osteomyelitis. J Korean Assoc Oral Maxillofac Surg 2020;46:19-27.

17. Nadella KR, Kodali RM, Guttikonda LK, Jonnalagadda A. Osteoradionecrosis of the jaws: clinico-therapeutic management: a literature review and update. J Maxillofac Oral Surg 2015;14:891901.

18. Lyons A, Ghazali N. Osteoradionecrosis of the jaws: current understanding of its pathophysiology and treatment. Br J Oral Maxillofac Surg 2008;46:653-60.

19. Straub JM, New J, Hamilton CD, Lominska C, Shnayder Y, Thomas SM. Radiation-induced fibrosis: mechanisms and implications for therapy. J Cancer Res Clin Oncol 2015;141:1985-94.

20. Stubblefield MD, Levine A, Custodio CM, Fitzpatrick T. The role of botulinum toxin type $A$ in the radiation fibrosis syndrome: a preliminary report. Arch Phys Med Rehabil 2008;89:417-21.

21. Ortigara GB, Schulz RE, Soldera EB, et al. Association between trismus and dysphagia-related quality of life in survivors of head and neck cancer in Brazil. Oral Surg Oral Med Oral Pathol Oral Radiol 2019;128:235-42.

22. Loorents V, Rosell J, Karlsson C, Lidback M, Hultman K, Borjeson S. Prophylactic training for the prevention of radiotherapy-induced trismus: a randomised study. Acta Oncol 2014;53:530-8.

23. Pauli N, Svensson U, Karlsson T, Finizia C. Exercise intervention for the treatment of trismus in head and neck cancer: a prospective two-year follow-up study. Acta Oncol 2016;55:686-92.

24. Kim A, Stubblefield MD. The role of the headmaster collar (Cervical) for dropped head syndrome in Hodgkin lymphoma survivors. PM R 2019;11:939-43.

25. Urken $M L$, Jacobson AS, Lazarus CL. Comprehensive approach to restoration of function in patients with radiation-induced pharyngoesophageal stenosis: report of 31 patients and proposal of new classification scheme. Head Neck 2012;34:1317-28.

26. Borrelli MR, Shen AH, Lee GK, Momeni A, Longaker MT, Wan DC. Radiation-induced skin fibrosis: pathogenesis, current treatment options, and emerging therapeutics. Ann Plast Surg 2019; 83:S59-64.

27. Bray FN, Simmons BJ, Wolfson AH, Nouri K. Acute and chronic cutaneous reactions to ionizing radiation therapy. Dermatol Ther (Heidelb) 2016;6:185-206.

28. Anand A, Balasubramanian D, Subramanian N, et al. Secondary lymphedema after head and neck cancer therapy: a review. Lym- 
phology 2018;51:109-18.

29. Boomsma MJ, Bijl HP, Langendijk JA. Radiation-induced hypothyroidism in head and neck cancer patients: a systematic review. Radiother Oncol 2011;99:1-5.

30. Stephen $A E_{1}$ Chen $K T$, Milas $M$, Siperstein AE. The coming of age of radiation-induced hyperparathyroidism: evolving patterns of thyroid and parathyroid disease after head and neck irradiation. Surgery 2004;136:1143-53.

31. Balentova S, Adamkov M. Molecular, cellular and functional effects of radiation-induced brain injury: a review. Int J Mol Sci 2015;16:27796-815.

32. Ko HC, Powers AR, Sheu RD, et al. Lhermitte's sign following VMAT-based head and neck radiation-insights into mechanism. PLoS One 2015;10:e0139448.

33. Cai Z, Li Y, Hu Z, Fu R, et al. Radiation-induced brachial plexopathy in patients with nasopharyngeal carcinoma: a retrospective study. Oncotarget 2016;7:18887-95.

34. Dimitrova A, Murchison C, Oken B. Acupuncture for the treatment of peripheral neuropathy: a systematic review and meta-analysis. J Altern Complement Med 2017;23:164-79.

35. Warade AC, Jha AK, Pattankar S, Desai K. Radiation-induced brachial plexus neuropathy: a review. Neurol India 2019;67:S47-52.

36. Reichstein D. Current treatments and preventive strategies for radiation retinopathy. Curr Opin Ophthalmol 2015;26:157-66.

37. Landier W. Ototoxicity and cancer therapy. Cancer 2016;122: 1647-58.

38. Nader ME, Gidley PW. Challenges of hearing rehabilitation after radiation and chemotherapy. J Neurol Surg B Skull Base 2019; 80:214-24.

39. Coumbe BG, Groarke JD. Cardiovascular autonomic dysfunction in patients with cancer. Curr Cardiol Rep 2018;20:69.

40. Mancuso M, Pasquali $E_{1}$ Leonardi $S$, et al. Oncogenic bystander radiation effects in Patched heterozygous mouse cerebellum. Version 2. Proc Natl Acad Sci U S A 2008;105:12445-50.

41. Shah-Becker S, Pennock M, Sinoway L, Goldenberg D, Goyal N.
Baroreceptor reflex failure: review of the literature and the potential impact on patients with head and neck cancer. Head Neck 2017;39:2135-41.

42. Arthurs E, Hanna TP, Zaza K, Peng Y, Hall SF. Stroke after radiation therapy for head and neck cancer: what is the risk? Int J Radiat Oncol Biol Phys 2016;96:589-96.

43.Grandhi R, Brasiliense LB, Williamson R, Zwagerman NT, Sauvageau $E$, Hanel RA. Delayed pipeline embolization of a ruptured true internal carotid artery aneurysm presenting with epistaxis: case report and review of the literature. World Neurosurg 2019;125:273-6.

44. Fernandez-Alvarez V, Lopez F, Suarez C, et al. Radiation-induced carotid artery lesions. Strahlenther Onkol 2018;194:699-710.

45. Giannopoulos S, Texakalidis P, Jonnalagadda AK, Karasavvidis T, Giannopoulos S, Kokkinidis DG. Revascularization of Radiation-Induced Carotid Artery Stenosis With Carotid Endarterectomy vs. Carotid Artery Stenting: A Systematic Review and Meta-Analysis. Cardiovasc Revasc Med 2018;19:638-44.

46. Briasoulis A, Silver A, Yano Y, Bakris GL. Orthostatic hypotension associated with baroreceptor dysfunction: treatment approaches. J Clin Hypertens (Greenwich) 2014;16:141-8.

47. Hayat A, Whittam D. Baroreceptor failure related to bilateral carotid artery disease: an uncommon cause of labile hypertension. Intern Med J 2014;44:105-6.

48. Mann SJ. Labile and paroxysmal hypertension: common clinical dilemmas in need of treatment studies. Curr Cardiol Rep 2015; 17:99.

49. Brook I. Paroxysmal hypertension episodes caused by direct massage of the carotid artery by ultrasound of the neck in a laryngectomee. J Med Ultrasound 2020;28:114-6.

50. Zhao W, Lei H, Zhu X, et al. The clinical characteristics of secondary primary tumors in patients with nasopharyngeal carcinoma after intensity-modulated radiotherapy: a retrospective analysis. Medicine (Baltimore) 2016;95:e5364. 